\author{
Classification \\ Physics Abstracts \\ 0.675-7.188
}

\title{
ION IMPLANTATION AND HYPERFINE INTERACTIONS IN METALS
}

\author{
H. BERNAS \\ Institut de Physique Nucléaire, BP 1, 91406 Orsay, France
}

(Reçu le 13 novembre 1973)

\begin{abstract}
Résumé. - Présentation simplifiée des techniques radioactives d'interactions hyperfines et application aux études d'implantation ionique dans les métaux. Quelques expériences caractéristiques. Etudes locales d'effets de dommages induits par l'implantation.

Abstract. - A very simplified description of angular distribution hyperfine interaction techniques is given, with a view to presenting their applications in ion-implantation studies of metals. Some typical experiments are reviewed. The latter part of the paper is devoted to a discussion of radiation damage effects in hyperfine interaction studies after ion-implantation.
\end{abstract}

This paper is a modified version of a talk originally presented at the Colloquium on Ion Implantation (Centennial Meeting of the French Physical Society, Vittel, May 1973). The purpose of the talk was primarily to convince radiation damage physicists that radioactive hyperfine interaction (hfi) techniques and results could be of considerable interest to their own work $\left(^{*}\right)$. A very condensed introduction to hfi methods was given, as well as some examples of hfi contributions to the physics of ion-implanted systems and a short discussion of information obtained on point defect (or cluster) - impurity interactions in metals. Because of its importance both to implantation - hfi experiments and to radiation damage analysis, the latter subject will be emphasized here ; the first two will only be briefly touched upon, mostly to indicate references.

1. Hyperfine interactions : What they are about. Let us first indicate how well-adapted the hfi methods are to implantation studies. Consider the main features of an implanted specimen : (i) in most cases, dilute alloys are studied, and the total number of implanted atoms is small typically $10^{11}-10^{15}$; (ii) during the slowing-down of the implanted ion, a large number ( $\gtrsim 10^{3}$ for a $100 \mathrm{keV}$-ion) of Frenkel pairs are produced in the host ; the interaction of defects, both among themselves and with the implanted impurities, is an important problem; (iii) when «low-energy »

(1) In fact, the talk was restricted to the « angular distribution 》 techniques (perturbed angular correlations and nuclear orientation); the Mössbauer effect technique was surveyed in a companion paper by G. Czjzek. $(\leqslant 500 \mathrm{keV})$ ion-implantors are used, implantation is a non-uniform surface effect (ion ranges are $\lesssim 1000 \AA$ ).

Table $I$ is an attempt to situate the radioactive hfi methods among the other ion implantation studies. The techniques are classified as " macroscopic » when they average over the whole sample, or " microscopic" when they probe only the immediate surroundings of the implanted impurity (except in the case of electron microscopy). They are termed " direct " if they give straightforward access to a property of the implanted impurity (e. g. the susceptibility of a magnetic impurity implanted in a diamagnetic host), and indirect otherwise (e. g., a resistivity experiment in which the contribution of the impurity is only obtained after deducing that of the host and defects).

Radioactive hfi techniques are " trigger-detection » methods : the nuclei of the implanted atoms alone send out information from the sample ; there is no input to the system and very "little " noise " from the host nuclei. The sensitivity is therefore very high. Moreover, as shown below, the hfi are essentially determined by the local properties of impurity interaction with neighbours (or defects) : hence, non-uniformity over the sample is not a drawback. Also, for this reason, it is often possible to identify different lattice sites occupied by the implanted ions, if their number is not too large (typically $\leqslant 3$ ).

Consider the effect of a static magnetic interaction on an impurity ion (spin $J$ ) in a solid. The effect of the applied field is to split the $\mid J>$-states into $\mid J, M_{J}>$ substates. The interaction $(A \mathbf{I} . \mathbf{J})$ between the electronic spin and its nuclear counterpart $I$ splits the nuclear levels into $\mid I, M_{I}>$-substates (hyperfine splitting). Transitions between these nuclear substates are stu- 


\section{TABLE I}

Attempt to classify various experimental methods used to study impurities and their interactions with host atoms and/or radiation damage. Standard abbreviations are used for the susceptibility $\chi$, the magnetization $m$, the resistivity $\rho . E P R=$ electron paramagnetic resonance ; $N M R=$ nuclear magnetic resonance ; $C E S R=$ conduction electron spin resonance ; $M E=$ Mössbauer effect ; $P A C=$ perturbed angular correlations ; NO = nuclear orientation.

\begin{tabular}{|c|c|c|c|}
\hline \multirow{3}{*}{ DIRECT } & \multirow[t]{2}{*}{ Macroscopic } & Microscopic & Information on \\
\hline & & $\begin{array}{l}\text { elastic neutron scatt. } \\
\text { EPR }\end{array}$ & impurity atoms \\
\hline & & $\begin{array}{l}\text { NMR, ME } \\
\text { PAC, NO }\end{array}$ & impurity nuclei \\
\hline \multirow{4}{*}{ INDIRECT } & $\begin{array}{l}\text { Small angle } X \text {-ray scatt. } \\
\text { channeling }\end{array}$ & electron microscopy & $\begin{array}{l}\text { defect (or impurity) clusters } \\
\text { impurity site in lattice }\end{array}$ \\
\hline & \multirow{3}{*}{$\begin{array}{l}\text { X-ray diffraction } \\
\rho \text {, electronic } C_{\mathrm{v}} \\
\text { Hall, effect, etc. }\end{array}$} & \multirow{2}{*}{$\begin{array}{l}\text { inelastic neutron scatt. } \\
\text { EPR } \\
\text { CESR }\end{array}$} & lattice order \\
\hline & & & host + impurity $(+$ defects $)$ \\
\hline & & $\begin{array}{l}\text { CESR } \\
\text { NMR, ME } \\
\text { nuclear } C\end{array}$ & host nuclei \\
\hline
\end{tabular}

died via hfi methods. In most cases, the amplitude of the nuclear splittings is not determined directly by the external perturbation : it is due to the polarization (deformation) of the electronic shells, via the magnetic part of the $A \mathbf{I}$.J. interaction. Similarly, the distortions of the electronic shell by any asymmetry of the electrostatic charge distribution around the impurity ion is reflected in the nuclear hyperfine splitting via the electric part of the interaction; electron spin relaxation álso produces transitions among nuclear sublevels. In most cases, the evolution of the impurity electron shells, even in metals, is determined to a great extent by their overlap with their neighbouring ions: this short-range interaction is the origin of the " microscopic » character of hfi measurements.

There are a number of excellent reviews on the theoretical and experimental aspects of the various radioactive hfi techniques (see, for instance [1]-[6]); the following is very much a bird's eye view of the principles.

In order to approach the radioactive hfi techniques, consider a transition between two different nuclear states, in the absence and in the presence of a splitting of the nuclear levels by some perturbation (Fig. 1). The relevant parameter is the energy difference between the nuclear substates. A direct measurement of this quantity can be made by comparison of the transition energy $E$ to the transition energies shown in the right-hand part of figure 1. This may be done, if the conditions for recoilless emission and absorption of $\gamma$-rays (Mössbauer effect) are satisfied, by a resonant absorption or scattering experiment in which the emitter is Doppler-shifted relative to the absorber (or vice versa) [2]. This differential energy measurement produces a spectrum such as that displayed at the bottom of figure 1 , in which e. g., the Larmor
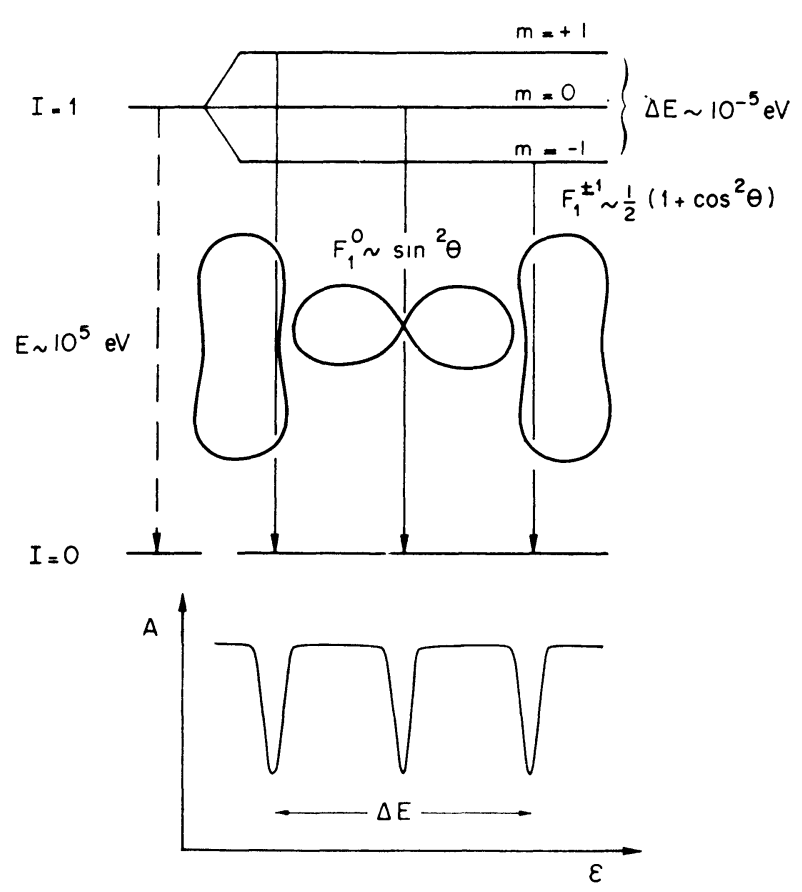

Fig. 1. - Splitting of an $I=1$ nuclear level by a magnetic hfi. Angular distributions of transition to $I=0$ ground state are shown : PAC and N. O. average over these. The Mössbauer effect «sees" the transitions from sublevels of the excited state to those of the ground state.

precession frequency is read off directly from the (equal) spacing of the absorption dips : $\delta E=h \omega_{\mathrm{L}}$. When the nuclear $g$-factor is known, the effective magnetic field at the nucleus may be deduced. In cases where the quadrupole moment of the nucleus interacts with the electric field gradient set up at the nuclear site by a non-spherical charge distribution around the impurity, the $+M_{I}$ and $-M_{I}$-sublevels are degene- 
rate. If a magnetic interaction is also present, more than one single transition frequency $\omega_{i}$ is found between the lines (the quadrupole interaction is very sensitive to the local symmetry around the impurity : it is therefore an excellent tool for ion implantation studies).

The hyperfine splitting cannot always be measured by such spectroscopic techniques, since the conditions for Mössbauer resonance are rather stringent [2]. A rather more widely applicable technique makes use of the anisotropy of the $\gamma$-ray emission. As shown in figure 1 for a very simple case, the angular distribution associated with each individual transition from a sublevel of the excited state to the ground state is anisotropic. Since the energy resolution is insufficient to distinguish the transitions, if all sublevels are equally populated the measured angular distribution is isotropic (see the sum $F_{1}^{0}+F^{ \pm 1}$ in figure 1). However, if by some means the excited nuclear sublevels can be unequally populated, knowledge of the nuclear parameters and geometrical properties of the radiation allows us to deduce the hyperfine splitting from the $\gamma$-ray anisotropy. Modifications in excited nuclear substate populations may be obtained by the various methods of nuclear orientation (N. O.) at very low temperature [3] (e. g., for a magnetic hfi, a Boltzmann distribution produces visible anisotropy when $\mu H / k T \sim 1$, i. e. when $T \sim 10 \mathrm{mK}$ for effective fields of order $\left.10^{5} \mathrm{Oe}\right)$. In such experiments, the thermal orientation is generally produced in the radioactive ground state of some isotope, and one studies the angular distribution of, e. g., $\alpha$ - or $\beta$-particles emitted in the desintegration, or of the $\gamma$-rays from the daughter nuclei. Unequal population of sublevels may also be produced in a perturbed angular correlation (P. A. C.) experiment [3]. The excited level depicted in figure 1 is then studied by a coincidence method : the detection of a particle (or $\gamma$-ray) preceding the level under study selects a quantization axis around which the angular distribution of the succeeding radiation is measured. In this case, there is no requirement on the temperature of the source. The form of the distribution $W(\theta)$ is quite similar in both cases :

$$
\begin{aligned}
& \text { N. O... } W(\theta)=1+\sum_{k} B_{k} U_{k} F_{k} P_{k}(\cos \theta) \\
& \text { P. A. C... } W(\theta)=1+\sum_{k} G_{k}(t) A_{k} P_{k}(\cos \theta)
\end{aligned}
$$

where $P_{k}(\cos \theta)$ is the Legendre polynomial of order $k$, and $U_{k} F_{k}$ and $A_{k}$ describe the geometrical properties of the nuclear radiations. The quantities $B_{k}$ and $G_{k}(t)$ are, respectively, the orientation parameter and the $P A C$ attenuation coefficient : in N. O., which is a static measurement, the degree of orientation increases when the magnetic hfi is increased; in P. A. C., a « time-window » is introduced by the lifetime of the intermediate nuclear state, and transitions among substates during this lifetime attenuate the anisotropy of the angular correlation due to the nuclear proper- ties. It is of interest to note that in a number of cases (e. g., magnetic hfi when $T$ decreases in N. O. ; « decoupling " experiments in P. A. C.), the limiting value of parameters $B_{k}$ and $G_{k}$ can be made equal to unity [3], hence that the orientation (or anisotropy) should be fully restored. This is often an important point to check after ion implantation, in order to make sure that the hfi acting on all nuclei in the sample are effectively accounted for.

The experimental techniques are schematically summarized in figure 2. For practical reasons, they may be placed under two headings. The " radioactive source " techniques are those which use standard nuclear spectroscopy techniques: they are welladapted to studies of long-lived radioactive isotopes,

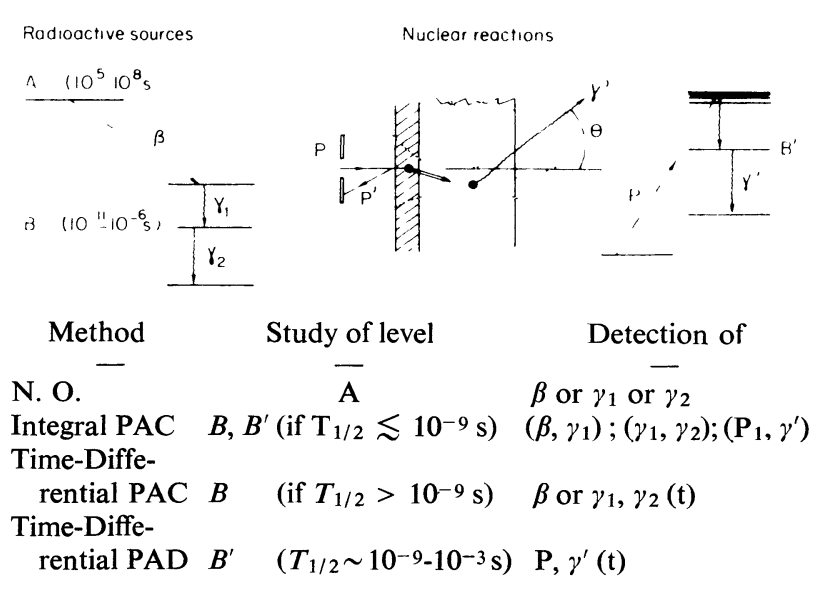

FIG. 2. - Summary of main angular distribution hfi techniques. Convenient lifetime ranges are indicated. The particle indicated $\beta$ is often an electron or a positron; it may also be a $\alpha$-particle. If the lifetime of the upper state in the daughter nucleus (left hand figure) is long enough to lead to reorientation, the hfi of this state may also be measured by N. O. In some cases, the combination of $\mathbf{N}$. O. with the Mössbauer effect can also provide interesting information both on the parent and on the daughter nucleus [13].

implanted via an isotope-separator (i. e. : at rather low energies) or - in some cases - via the nuclear reaction producing the radioactive species itself (in most cases, this entails high-energy self-implantation). The angular distribution anisotropies involved here are not very large (less than $50 \%$ ), but these techniques are particularly useful for systematic studies in which some external parameter (e. g. the implanted alloy's temperature) must be carefully monitored. The " accelerator beam » techniques are those in which implantation is due to the recoil energy associated with the nuclear reaction that produces the excited state under study. For example [4], [6], a thin coating of the element to be studied is deposited on the prospective host ; a Coulomb excitation process via a high-energy particle (e. g. $30 \mathrm{MeV}$ Oxygen ions) both excites the desired nuclear level and implants the impurity into the host. High-energy implantation is the general rule here, but recoil energies as low as $100 \mathrm{keV}$ have also been 
used [6]. The in-beam experiments have two specific features. First, there is generally no long-lived parent of the nuclear state under concern : since the reaction and slowing-down times are very short $\left(\sim 10^{-12} \mathrm{~s}\right)$, all the interactions acting on the implanted impurity are turned on during the lifetime of the state. If the lifetime is short enough ( $\left.\lesssim 5 \times 10^{-11} \mathrm{~s}\right)$, the impurity may not even have stopped when the state decays: this leads to the very interesting " transient hf field" problem [9]. The second feature is that very large anisotropies are observed in the angular distributions after nuclear reactions (up to factors of 10) ; relevant data may therefore be collected on very low-dose implants (less than $10^{10}$ atoms $/ \mathrm{cm}^{2}$ ).

The time-ranges which may be conveniently studied by the various techniques are indicated in figure 2 . The discrimination between " integral » and " differential » $\mathrm{PAC}$ (or PAD) is related to the experimental time resolution $T_{\mathrm{R}}$. Consider an interaction (such as the magnetic hfi) whose characteristic time $T_{\mathrm{i}}$ (e. g. Larmor period) is of the order of the nuclear lifetime $\tau_{\mathrm{N}}$.If $T_{\mathrm{R}} \gg \tau_{\mathrm{N}}$, the experiment integrates over the time-evolution of the hfi during the lifetime $\tau_{\mathrm{N}}$ : only an average is seen. It is easily shown, for example, that for a magnetic hfi, this leads to a rotation as well as an attenuation of the PAC pattern shown in figure 2 and that the rotation is directly related to the Larmor frequency. By nature, this experiment averages over all the hfi acting on all the radioactive nuclei in the sample ; care must be exercised in the interpretation, since different hfi may act on inequivalent sites. This is also true for a N. O. experiment.

If $T_{\mathrm{R}}<\tau_{\mathrm{N}}$, the details of the time-evolution of the hfi may be studied. In the case of a static magnetic hfi, the angular correlation pattern shown in figure 2 may be seen to rotate during the nuclear lifetime : this appears as a periodic oscillation superimposed on the exponential decay of the state (Fig. 3). Characteristic oscillations also appear for a static electric quadrupole interaction, as shown in figure 4. Elaborate studies of angular correlation pattern behaviour in the presence of combined static electric and magnetic interactions have also been accomplished [3]. Since the time-evolution

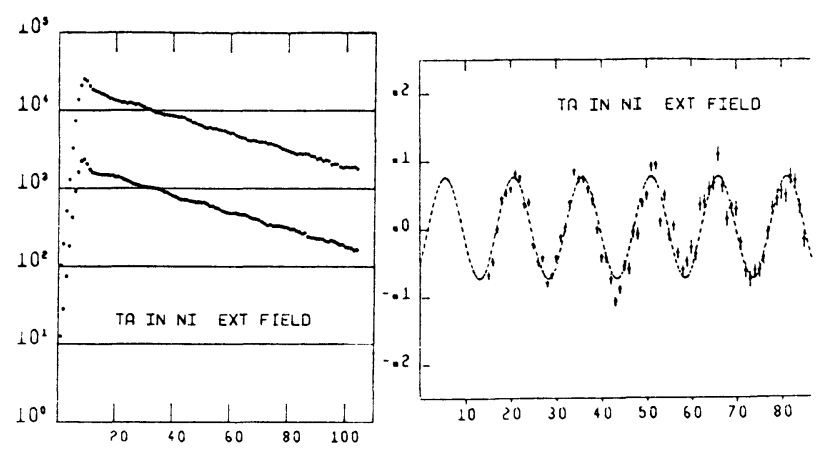

FIG. 3. - TDPAC measurement of static magnetic hfi for ${ }_{181} \mathrm{Ta}$ implanted into $\mathrm{Ni}[15]$. The Larmor precession of the angular correlation modulates the exponential decay of the nuclear state (left). The two counters are at a fixed angle of $135^{\circ}$.

On the right, the exponential decay has been removed.

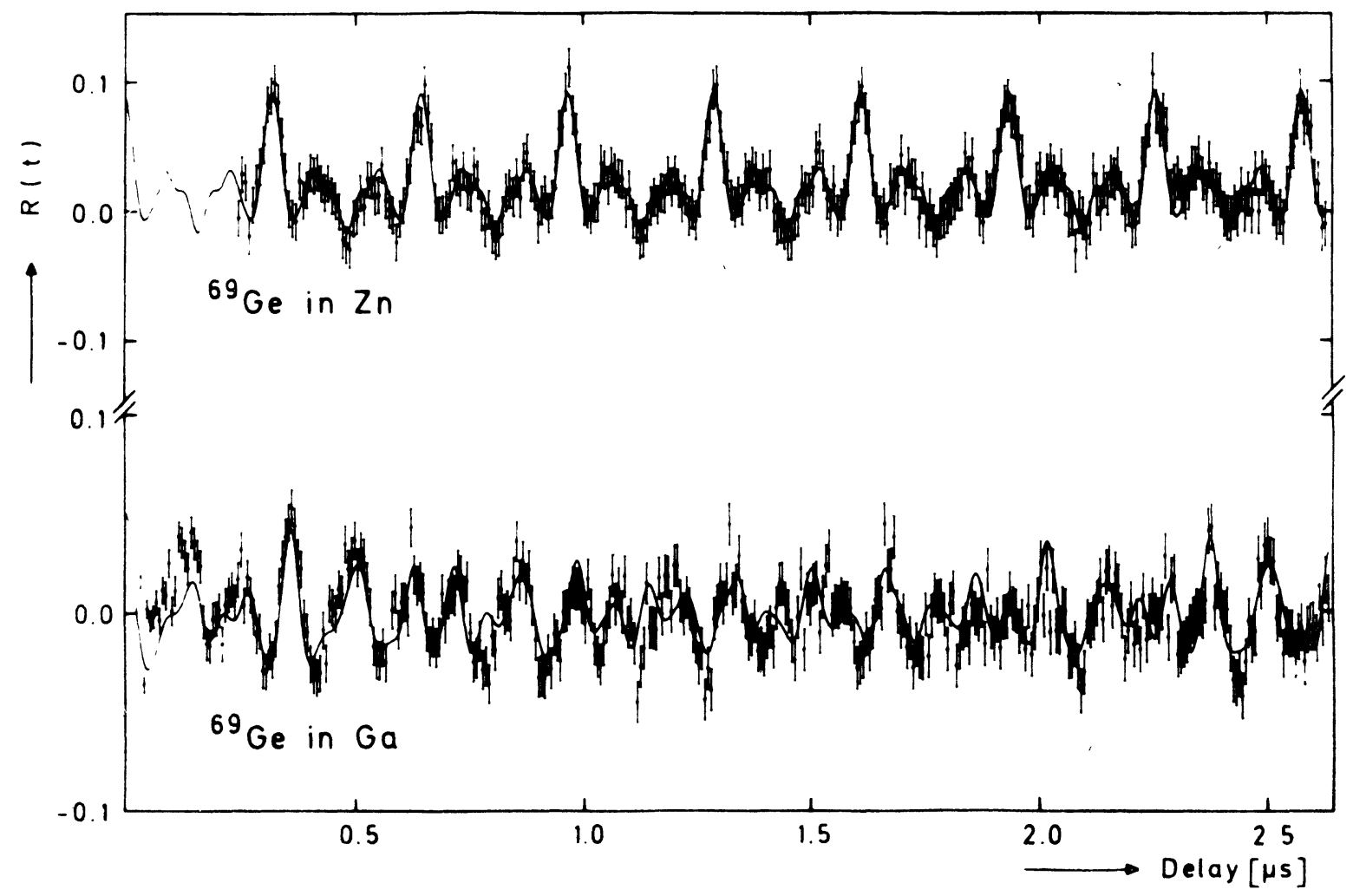

Fig. 4. - In-beam TDPAC measurement of static quadrupole interaction for ${ }^{69} \mathrm{Ge}$ in hcp $\mathrm{Zn}$ and orthorhombic Ga [16]. In $\mathrm{Zn}$, the EFG is axial; in $\mathrm{Ga}$, it is not. The counters are at a fixed angle $\left(90^{\circ}\right)$ and these curves appear as modulations of the nuclear state decay. 
of the various interactions is quite different, a DPAC measurement may distinguish between the hfi acting on different sites. The precision is then comparable to that obtained in Mössbauer effect experiments ; as in that case, the detailed analysis usually depends on whatever complementary information may be obtained on the system (e. g., lattice-location measurements, possible impurity-defect associations, etc...).

The effect of the various possible relaxation processes on radioactivity hfi experiments has also been studied in the cases where the electron relaxation time $T_{\mathrm{i}}$ is longer, shorter, or in the same range as $\tau_{\mathrm{N}}[10]$, thus establishing both the Mössbauer effect and PAC techniques as complementary to EPR and NMR [11]. The PAC method may be of particular advantage, since the only temperature dependence in an angular correlation experiment is that of the hfi. The sensitivity depends mainly on the ratio of the relaxation time to $\tau_{\mathrm{N}}$ : in many cases, relaxation may be studied over a time-range of some three orders of magnitude and over the corresponding temperature-range (Fig. 5).

2. Some typical applications to ion-implanted systems. - This paragraph is restricted to examples from angular distribution measurements : Mössbauer effect results are discussed in Ref. [12] and in the paper by G. Czjzek at this Conference. Problems related to implantation-induced radiation damage often appear in these experiments : they will be discussed in the next section.

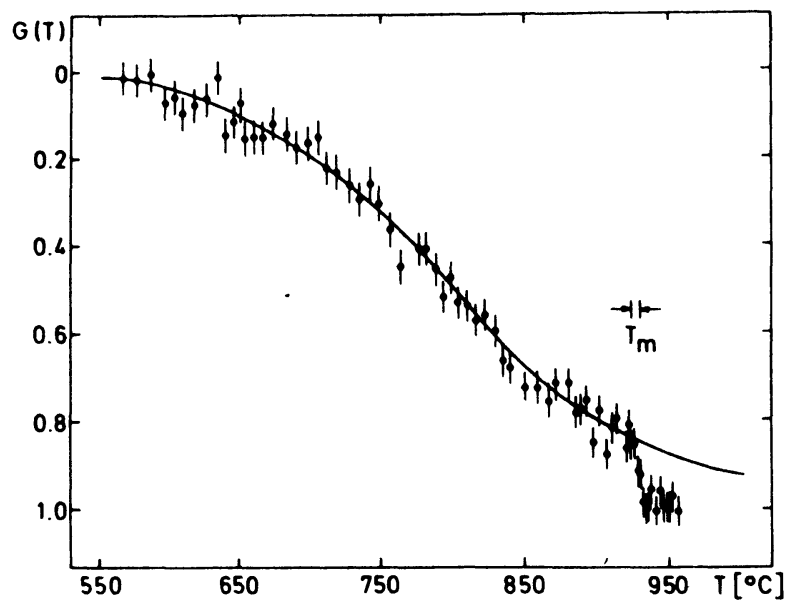

FIG. 5. - In-beam TDPAD measurement of relaxation for ${ }^{73} \mathrm{As}$ in $\mathrm{Ge}$ [17]. Note transition at melting-point $T_{\mathrm{m}}$.

The hyperfine hamiltonian always contains the product of a nuclear parameter (nuclear $g$-factor, quadrupole moment) by a quantity that characterizes the atomic environment (e. g., the effective magnetic field at the nucleus, the electric field gradient). Information from nuclear physics is therefore clearly required in order to interpret results on hif experiments. Conversely, knowledge of the large hyperfine fields acting at nuclei [1] has led to the measurement of a vast number of static nuclear moments (particularly of short-lived states) and to important developments in nuclear physics ; this has, in fact, been one of the main applications-in which " accelerator beam " implantation is essential-of hfi physics. Recent work in this field is summarized in Ref. [6] and [13], as well as in a Colloquium of this Meeting [14]. Here, we are concerned with the solid-state studies of unstable alloys prepared by implantation.

The largest activity in the field so far has been devoted to the measurement of magnetic hyperfine fields acting on nuclei in different metallic environments. For $d$ transition elements, this quantity is directly related to the spin polarization of the implanted impurity ; proper understanding of the relation between the hyperfine field and the susceptibility leads to valuable information on the s-d interaction in metals (see J. Flouquet, Ref [3]). The study of sp-impurities in ferromagnets has been an important source of information on conduction electron polarization. Ref. [4], [7] and [12] contain reports of many implantation-hfi experiments performed on such systems, in cases where standard metallurgical techniques are inoperative. A typical static hfi result from a TDPAC experiment on an implanted source is shown in figure 3 [15]. Figure 4 represents an accelerator-beam TDPAC study of the static electric quadrupole hfi acting on ${ }^{69} \mathrm{Ge}$ nuclei in an electric - field gradient (EFG) with or without axial symmetry. The work described in Ref. [16] yielded two nuclear quadrupole-moments and EFG ratios and the asymmetry parameter of the EFG tensor for orthorhombic Ga. The existence of a unique EFG and the absence of any time-dependent effects over a time-scale of several microseconds, as well as a comparison of the results to nuclear quadrupole resonance results on stable $\mathrm{Ga}$ isotopes, indicates that in these experiments (performed at temperatures $50^{\circ}$ below the meting points), the recoil ions end up in substitutional lattice sites. The impressive possibilities of angular distribution techniques for studying time-dependent phenomena are demonstrated [17] (Fig. 5) by a similar TDPAD experiment on ${ }^{73} \mathrm{As}$ produced from - and recoiled into - Ge. A pulsedbeam " stroboscopic " method [6] was used ; resonance curves were obtained, and their amplitude measured at various temperatures. The temperature-dependence of the attenuation coefficient $G(T)$ in figure 5 is accounted for by assuming a time-dependent perturbation of the lattice immediately surrounding the recoil ion; the unique activation energy $E$ of this process is in good agreement with values expected for vacancy or divacancy migration in Ge. Also, a remarkable combination of PAD and NMR techniques [18] has produced very high-accuracy magnetic and electric hfi results.

The advantages of ion implantation are particularly clear in the case of rare earth elements. Because of the small radius of the $4 \mathrm{f}$-shell, their ionic magnetic properties should be retained in metals : a hfi essentially due to the orbital contribution and a well-localized 
moment are expected. Strong L-S coupling also leads to a weak crystalline electric field. For magnetism studies, it is thus very tempting to produce, e. g., Fe-rare earth alloys. But because of their large ionic diameter (> $3 \AA$ ), rare earth ions are insoluble in such elements as $\mathrm{Fe}, \mathrm{Ni}, \mathrm{Cu}$; in gold, their solubility is negligible or very small for elements of the first half of the series, and reaches $8 \%$ for $\mathrm{Yb}$. The first attempt at such unstable metallic alloy production used a nuclear reaction (Coulomb excitation) [19] and the on-line IPAC technique ; in later work [20] using isotope-separator implanted ${ }^{169} \mathrm{Tm}$ in $\mathrm{Fe}$, the existence of a sharply localized moment was demonstrated (Fig. 6). The relaxation properties of rare earth ions, implanted in magnetic as well as non-magnetic hosts, have also been studied; in cases where comparison is possible with EPR experiments on alloys produced by classical metallurgy, reasonable agreement is found [21].

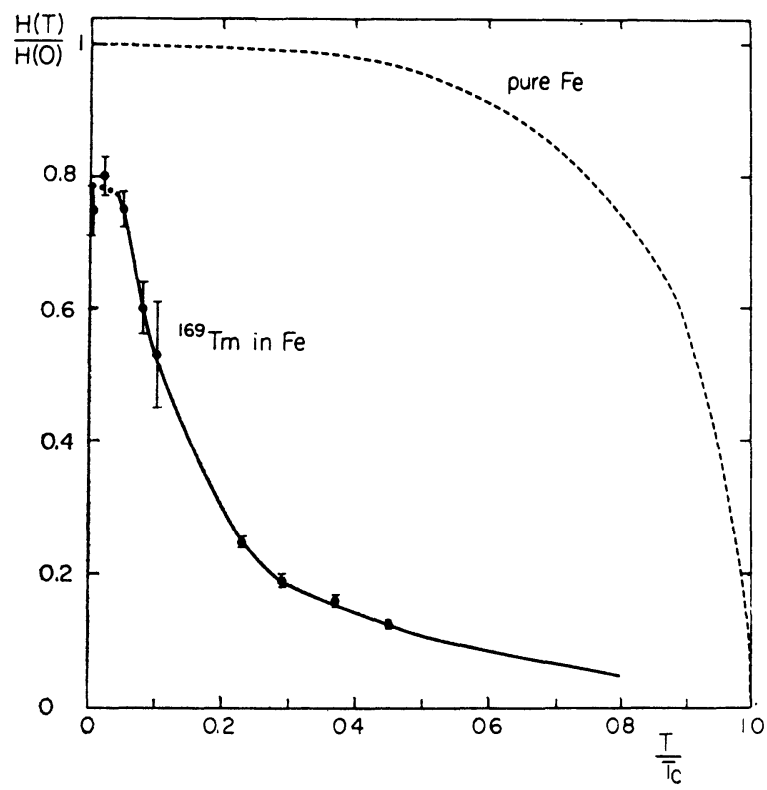

Fig. 6. - Radioactive source measurement of magnetic hfi temperature-dependence for ${ }^{169} \mathrm{Tm}$ in $\mathrm{Fe}$ [20]. The dotted curve is the magnetization curve of pure Fe. The $\mathrm{Tm}^{3+}$ ion behaves like a paramagnetic spin in an exchange field of $2.5 \mathrm{MOe}$ (electronic relaxation is also observed and accounted for).

As mentioned previously, when ion implantation and radioactivity hi techniques alone apply to the study of a system, considerable care must be exercised before concluding on the relative amplitude of the interactions experienced by the nuclei. This is rather clear from a N. O. experiment on $\mathrm{Fe}^{175} \mathrm{Yb}$ and $\mathrm{Au}^{175} \mathrm{Yb}$ (Fig. 7) [22], in which the sign of the anisotropy was found to differ for the two hosts. A pure magnetic interaction is expected to produce a negative anisotropy for the $396 \mathrm{keV} \gamma$-ray: this was indeed observed for ${ }^{175} \mathrm{Yb}$ in $\mathrm{Au}$ and a value of the ${ }^{175} \mathrm{Yb}$ ground state nuclear moment was deduced. The positive anisotropy observed for ${ }^{175} \mathrm{Yb}$ in $\mathrm{Fe}$ can only be explained in terms of a combined (magnetic + electric

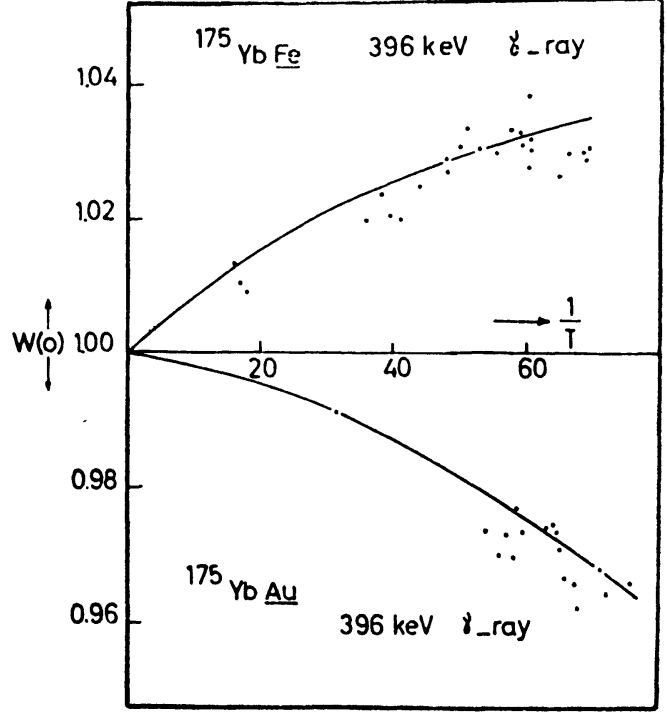

FIG. 7. - Nuclear orientation anisotropy measurements on ${ }_{175} \mathrm{Yb}$ in $\mathrm{Au}$ and $\mathrm{Fe}$ [22]. Note opposite variations of $\mathrm{W}(0)$ : for discussion, see text $\left(T\right.$ is source temperature, i. e. : $\left.\sim 10^{-2} \mathrm{~K}\right)$.

quadrupole) interaction [3]. This could indicate that the local symmetry of $\mathrm{Yb}$ in (cubic) $\mathrm{Fe}$ is non-cubic, due perhaps to some short-range impurity-defect interaction, in agreement with the discussion of de Waard [12], according to which large non-soluble impurities are expected to give rise to such effects, as opposed to cases (such as $\mathrm{Yb}$ in $\mathrm{Au}$ ) where the implanted impurity is reasonably soluble in the host. However, the situation is actually more complicated, as will be seen in the next paragraph.

3. Interactions between implanted impurities and defects. - It is quite clear to all experimentalists in the field today that when a specimen is prepared by ion-implantation, the outcome is actually a rather unstable ternary alloy whose components are the host, the desired dilute impurity, and the lattice defects - produced by the implantation - who are stable at the implantation temperature. Both calculations from simple kinematics and electronmicroscopy experiments show that the slowing-down of each incoming ion creates typically several thousand Frenkel pairs : thus alloys whose impurity content is deemed low (e. g., $10^{12}$ ions $/ \mathrm{cm}^{2}$ ) may contain high average concentrations of point defects $(\gtrsim 1 \%)$ in the implanted region, and the effect on the hfi may be rather drastic. The interactions between the host, impurities, and defects must therefore be studied in order to separate out the hfi behaviour of the impurity alone. The main questions are indicated in table II, along with the combination of standard macroscopic and microscopic methods (see Ref. [23]-[25]) that may provide answers to each of them. From recent work (including the above-mentioned references), it appears that fair knowledge of all the elements of this $(2 \times 2)$ matrix is needed to solve our problem. The studies 


\section{TABLE II}

Post-Implantation Questions and Clues to Answers. ("Lattice Location " refers to the association of the channeling technique with Rutherford scattering from the implanted impurity, as described in Ref. [25].)

HFI

$\begin{array}{ll}\text { LATTICE LOCATION } & \text { IMPURITY - } \\ \text { RESISTIVITY } & \text { Final Site(s) ? } \\ & \text { Existence (Nature) of } \\ \text { Impurity. Defect Complex ? }\end{array}$

\section{ELECTRON MICROSCOPY}

Symmetry of Defects (Clusters) and of Impurity. Defect Complex ? STABLE DEFECTS (Clusters) ? represented by the diagonal elements characterize " pure " defect or impurity properties at the implantation temperature; off-diagonal elements connect static impurity and defect properties to each other, and therefore depend on prior knowledge of the diagonal elements. Fortunately, a wealth of information is at hand, thanks to the work of radiation damage physicists, and implantation hfi experiments are benefiting increasingly from it. The feedback has started also, as shown by very recent hfi and lattice - location experiments that shed new light on old radiation damage problems.

Without any claim to completeness, and with due apologies for a heavily biased selection, let us now consider a few (hopefully significant) examples : we wish to show that (i) impurity-defect interactions definitely do affect hfi experiments, and (ii) the combination of hfi experiments with the other methods of table II provides new quantitative information on these interactions. Our first cases concern implantations into non-magnetic hosts.

As noted above, although the N. O. experiment [22] on ${ }^{175} \mathrm{Yb}$ in Fe certainly indicated a post-implantation difficulty (we shall come back to that later), all was apparently well with the implanted $\mathrm{Au}^{175} \mathrm{Yb}$ alloy. Recently, however, the latter N. O. experiment has been re-done : in one case [26], it was performed both on the as-implanted alloy and (taking advantage of the high solubility) on the same source after melting; in the other case [27] only the melted alloy was studied. The rather striking result of [26] is shown in figure 8 , which presents the magnetization curves for the sample before and after melting : the lower curve agrees with the result of [21], while the high-field limit of $\left\langle J_{\mathbf{Z}}\right\rangle$ is seen to be significantly higher after melting (the value is essentially in agreement with the result of [27]). This indicates that the $\mathrm{Yb}$ ions were not properly dissolved in state $A$. For example, if a fraction $f$ of the $\mathrm{Yb}$ population were fully aligned at high field (with $H_{\mathrm{hf}}$ given by curve B) and all the rest were pinned down in other directions, the number $f$ would only be about 0.35 . Clearly, the solubility of $\mathrm{Yb}$ in $\mathrm{Au}$ is not a sufficient criterion to preclude radiation damage problems.

Some time ago, the nature of the damage was studied by electron microscopy for $\mathrm{AuYb}$ and $\mathrm{FeYb}$ [28] :

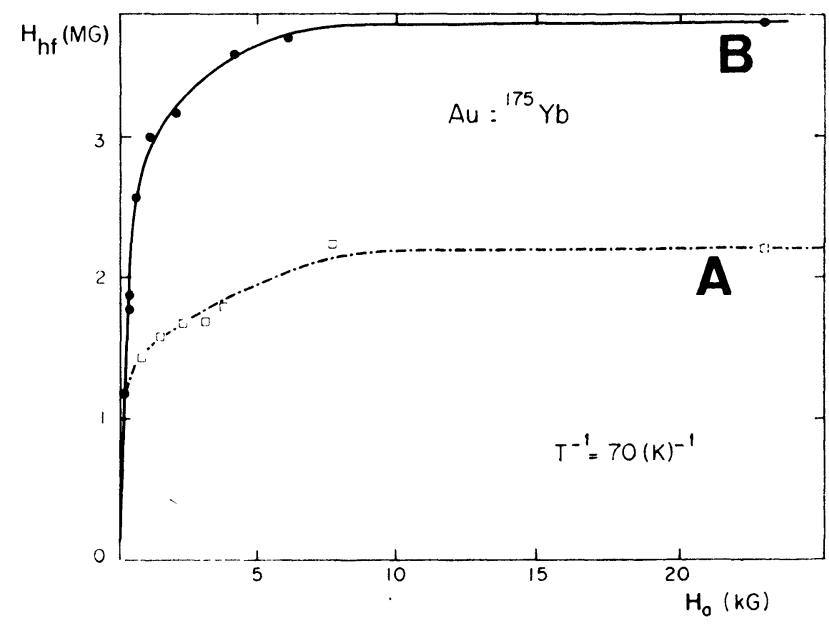

FIG. 8. - Nuclear orientation measurement of the ${ }^{175} \mathrm{Yb}$ spin magnetization in $\mathrm{Au}$, in applied fields $H_{\mathrm{A}}$ [26]. For discussion, see text.

using standard stereoscopy techniques and contrast rules, stable defect clusters were identified as vacancy loops in both cases ; their depth distribution was measur ed, and shown not to overlap with the implanted ion distribution (Fig. 9). From the result on $\mathrm{AuYb}$, it was suggested that about one-third of the $\mathrm{Yb}$ ions were in a

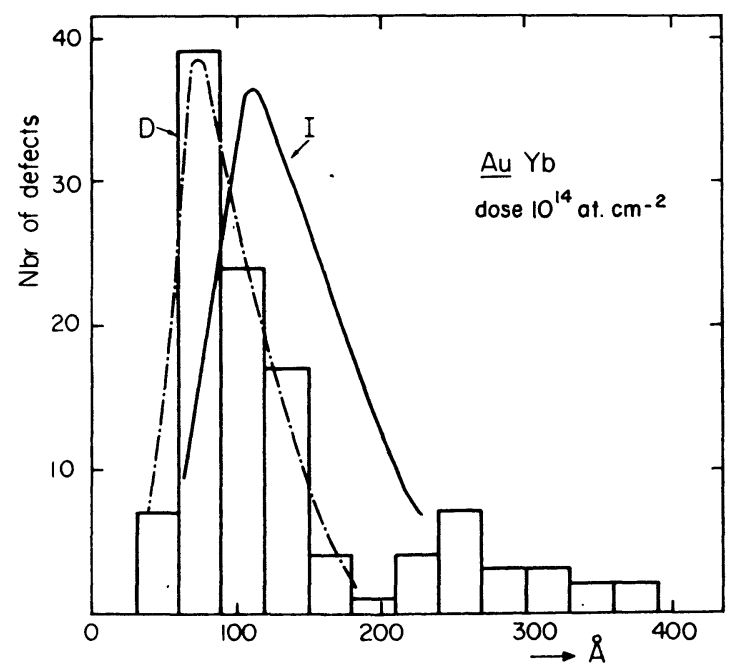

FIG. 9. - Electron microscopy measurement of depth distribution of radiation damage-induced vacancy-loops in AuYb [28] (Curve D) compared to implanted ion distribution (curve I). 
proper « dilute alloy », while the others were likely to interact with the large concentration of defects nearer to the surface, and possibly « precipitate» (i. e. : form Yb clusters or $\mathrm{Au}-\mathrm{Yb}$ intermetallic compounds). Since - as shown by resistivity measurements [23] - an annealing stage for vacancies in gold sets in just below room temperature, "precipitation " may be assisted by the existence of a cloud of vacancies in the vicinity of the impurity. The agreement between the result of [26] and the value predicted for $f$ in [28] may of course be fortuitous, but it does suggest that further work in this direction is indicated. In recent electron microscopy experiments on six elements ( $\mathrm{Ar}, \mathrm{Fe}, \mathrm{Kr}, \mathrm{Mo}, \mathrm{Xe}, \mathrm{Yb}$ ) implanted in $\mathrm{Au}$ [28b], the nature of the observed damage depends on the mass of the implanted species : interstitial loops are found for ions lighter than Mo, and vacancy loops are found for Mo and heavier masses. This result is not yet well understood: it could possibly be related to the probability of the light impurity ending up in interstitial positions and stabilizing interstitial $\mathrm{Au}$ atoms. If such were the case, hfi experiments would obviously be affected. This work is presently being extended.

The result on AuYb leads us to the following remark : if defects play an instrumental role in the stability of an implanted alloy, their evolution (i. e. : annealing stages) should determine that of the alloy. The features of this evolution depend on the impurity content of the host before implantation, and may depend somewhat on the implanted impurity, but if the only stable defects at the implantation temperature are vacancies and vacancy clusters, interesting information may be obtained from annealing studies of quenched - in vacancies in the pure host, whose results are usually available. This optimistic approach is encouraged by the following results.

In a 1962 study of vacancies in pure Ag quenched from high temperatures [29], a threefold drop in the resistivity between $238 \mathrm{~K}$ and $350 \mathrm{~K}$ (Fig. 10) was convincingly ascribed to the mobility of divacancies ; also, some evidence was found for the trapping of divacancies by impurities. Recently, a TDPAC expe-

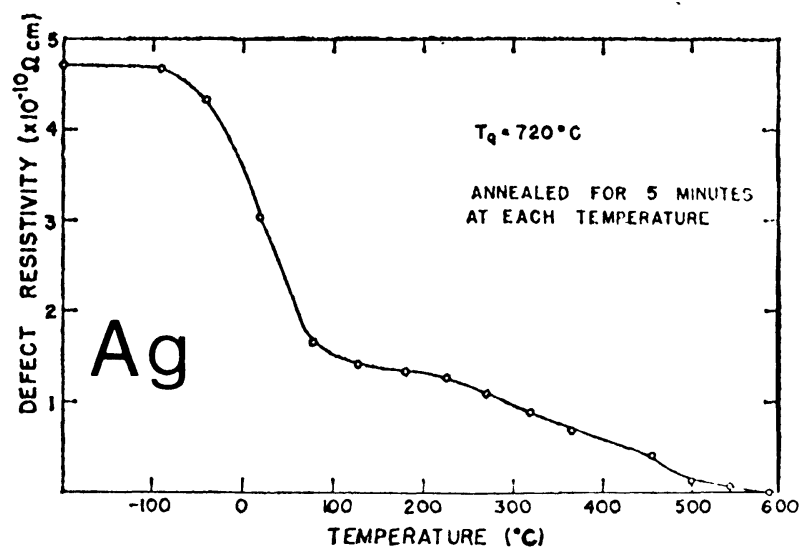

FIG. 10. - Temperature-dependence of defect resistivity of $\mathrm{Ag}$ quenched from high temperatures $\left(720^{\circ}\right)$ [29]. Note annealing stage around $0^{\circ} \mathrm{C}$. riment was performed [30] on ${ }^{111}$ In-implanted $\mathrm{Ag}$ (via the $(\alpha, 2 \mathrm{n})$ reaction in situ : since ${ }^{111} \mathrm{In}$ decays with a 2.3 day half-life, this is actually a « radioactive source " experiment). Two different hfi populations were found immediately after implantation for the ${ }^{111} \mathrm{Cd}$ daughter in (cubic) Ag, both of which involved an electric quadrupole interaction (Fig. 11) : again,

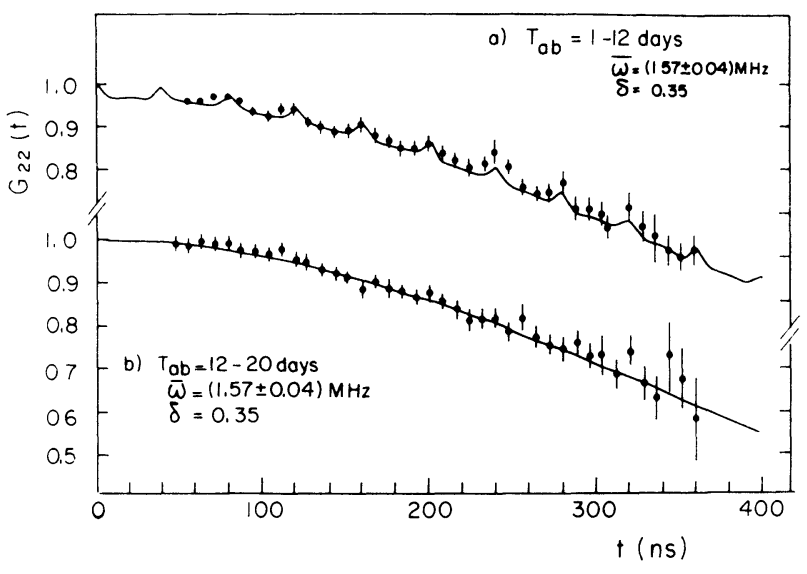

Fig. 11. - Quadrupole hfi TDPAC curves for ${ }^{111} \mathrm{Cd}$ recoiled into $\mathrm{Ag}$ [30]. $\mathrm{T}_{\mathrm{a}}$ is time after irradiation. The superposition of two different quadrupole interactions is clearly seen in the upper curve, as well as the disappearance of the high-frequency component after $\sim 12$ days (see text).

in spite of the high solubility of In in Ag, the implanted ions are not simply substitutional at room temperature. One species (abundance $\sim 97 \%$ ) involved a distribution of quadrupole interaction (width $\sim 35 \%$ ) around a weak average frequency - i. e. : a weak EFG and did not change with time while the remaining $3 \%$ displayed a single, strong $(\sim 100 \mathrm{MHz})$ quadrupole interaction which disappeared after a few days. Since the time-evolution observed in this TDPAC experiment corresponds rather well to the temperaturedependence found in [29], it is tempting to speculate that the sharp $(100 \mathrm{MHz})$ interaction corresponds to ${ }^{111} \mathrm{Cd}$ atoms associated to divacancies, while the spread of interactions experienced by the majority of the impurities is a consequence of their associating with (and clustering into ?) the vacancy loops formed by divacancy clustering during this annealing stage. Therefore, if this interpretation were correct, one would expect the " high frequency interaction" component to dominate if the implantation and measurements were carried out at temperatures below the annealing stage: such an experiment would be of considerable interest $*$. Note the advantage of

* Note added in proof : This experiment has now been performed. The $(\alpha, 2 n)$ reaction implantations were carried out at $80 \mathrm{~K}$, and TDPAC spectra were measured at $80 \mathrm{~K}$ after anneals at temperatures up to $350 \mathrm{~K}$. Marked differences are indeed observed between the various TDPAC spectra; however it is not clear that the simple description in terms of «low» and " high frequency " components still holds. (SAGE, F., ThOmé, L., Do NGOC, B. and Bernas, H., submitted to Int. Conf. on Hyperfine Interactions in Nucl. React. and Decay, Uppsala, June 1974). 
the TDPAC technique here, since different sites are readily distinguished. Along the same lines, albeit outside the scope of this paper, two relevant Mössbauer experiments should at least be mentioned, involving a study of quenched AlFe [31] in which the interaction of vacancy loops with $\mathrm{Fe}$ impurities is considered, and an elegant low-temperature study of neutron-irradiated $\mathrm{Al}^{57} \mathrm{Co}$ [32] which provides strong evidence of interstitial trapping by the impurities.

Now what about ferromagnetic implanted alloys ? This is clearly the area in which most experimental work has been carried out so far, in efforts to measure nuclear $g$-factors and magnetic hfi. The direct sensitivity of such measurements to environmental differences has often proved to be low - in many ways, this is rather fortunate... The quadrupole interaction, though more immediately dependent on the local symmetry of the impurity, is generally far weaker than its magnetic counterpart and hence far more difficult to measure when both coexist (this statement does not hold for a spectroscopic technique such as the Mössbauer effect). For dilute rare earths in, e. g., Fe the exchange field often dominates the CEF in the impurity hamiltonian [20], so that a « quasi-free ion » behaviour prevails : this is, for example, the case for FeDy [12] and FeTm [20]. An exception is $\mathrm{Yb}$, for which the CEF parameters are large enough for the CEF to overpower the exchange contribution and allow the large quadrupole interaction of ${ }^{175} \mathrm{Yb}$ (whose ground state quadrupole moment is a big 3 barns) to reveal itself, either because of the CEF ground state anisotropy, or because of some local distortion due to an impurity-defect interaction. Hence, the experiments on ${ }^{169} \mathrm{Tm}$ (parent ${ }^{169} \mathrm{Yb}$ ) and ${ }^{175} \mathrm{Yb}$ in $\mathrm{Fe}$ [20] [22] are not sensitive to the same effects.

Studies in ferromagnetic hosts have been used to obtain interesting information on the evolution of impurity-damage interactions-thus confirming the existence of such interactions. To our knowledge, the most complete work so far is that on $\mathrm{Fe} \mathrm{Yb}$, for which electron microscopy, hfi, and lattice location experiments were performed at various annealing temperatures. From the electron microscopy experiments [28], it was concluded that the vacancy loops lie in the $\{100\}$ planes ; moreover, it is well-known [24] that their diameter increases with increasingly high annealing temperatures, until they release vacancies and anneal out. The strong correlation of the latter process with the changes in the measured average magnetic hfi at ${ }^{169} \mathrm{Tm}$ and in the $\mathrm{Yb}$ backscattering yield from lattice-location experiments is shown in figure 12 [33], which also shows that the impurity evolution takes place in the $\{100\}$ plane. These results point to a rather detailed view of the process : apparently, the impurities interact with the vacancy loops in their planes (i. e. $\{100\}$ ) ; the mobility of the vacancies and of the impurities leads to $\mathrm{Yb}$ «precipitation " (clustering or intermetallic compound for- mation), as demonstrated by the drop in the measured hfi, and in these " precipitates " (or clusters), at least $20 \%$ of the $\mathrm{Yb}$ atoms remain substitutional $\left({ }^{2}\right)$. The " precipitation " hypothesis is corroborated by a TDPAC experiment (Thomé, L. and Bernas, H., unpublished) on the $36 \mathrm{~ns}, 379 \mathrm{keV}$-state of ${ }^{169} \mathrm{Tm}$ in $\mathrm{Fe}$ after annealing at $550^{\circ} \mathrm{C}$ (i. e. : above the drop in $H_{\mathrm{hf}}$ ) : the ${ }^{169} \mathrm{Tm}$ nuclei were found (Fig. 13) to exhibit a distribution (width $\sim 15 \%$ ) of static quadrupole interactions centered around an average value of $260 \pm 80 \mathrm{MHz}$.

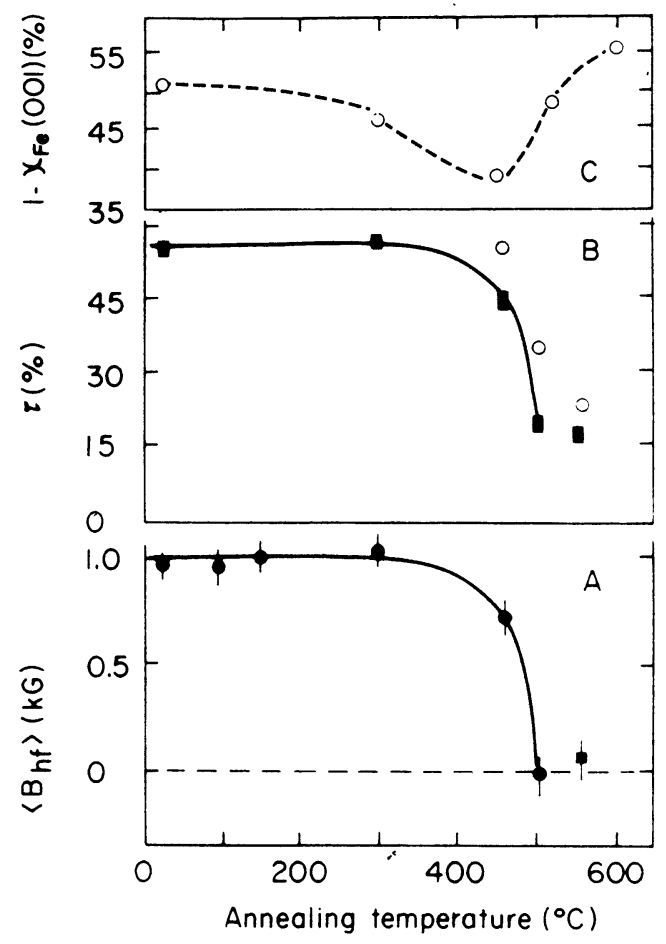

FIG. 12. - Annealing properties of Yb-implanted Fe [33]. Curve A : average magnetic hfi at ${ }^{169} \mathrm{Tm}$ nucleus. Values are normalized to -1760 MOe. Curve B : Corrected $\mathrm{Yb}$ extinction ratio (squares refer to all directions except (001) plane ; circles refer to that plane). Curve $\mathrm{C}$ : (001)-plane extinction ratio for the $\mathrm{Fe}$ host atoms at $\mathrm{Yb}$ implantation depth.

Further evidence for these radiation damage-impurity interactions is provided by the following results : (i) the transition temperature in figure 12 is in good agreement with a resistivity annealing stage $V$ measurement on quenched $\mathrm{Fe}$ containing impurities [34], and in reasonable agreement with that found in a latticelocation experiment on implanted FeBi [35]; (ii) an identical hfi experiment on $\mathrm{Ni}^{169} \mathrm{Yb}$ [33] yielded the same curve with a transition temperature of $550^{\circ} \mathrm{C}$, in

(2) The lattice-location experiments also show that there is no simple correlation between the back-scattering yield and the "substitutional fraction", and this is confirmed by the hfi work. Hopefully, the combination of the two techniques and electron microscopy will lead to a better understanding of how the «lattice-location» method really probes an implanted impurity site (effects of impurity size, radiation damage...). 

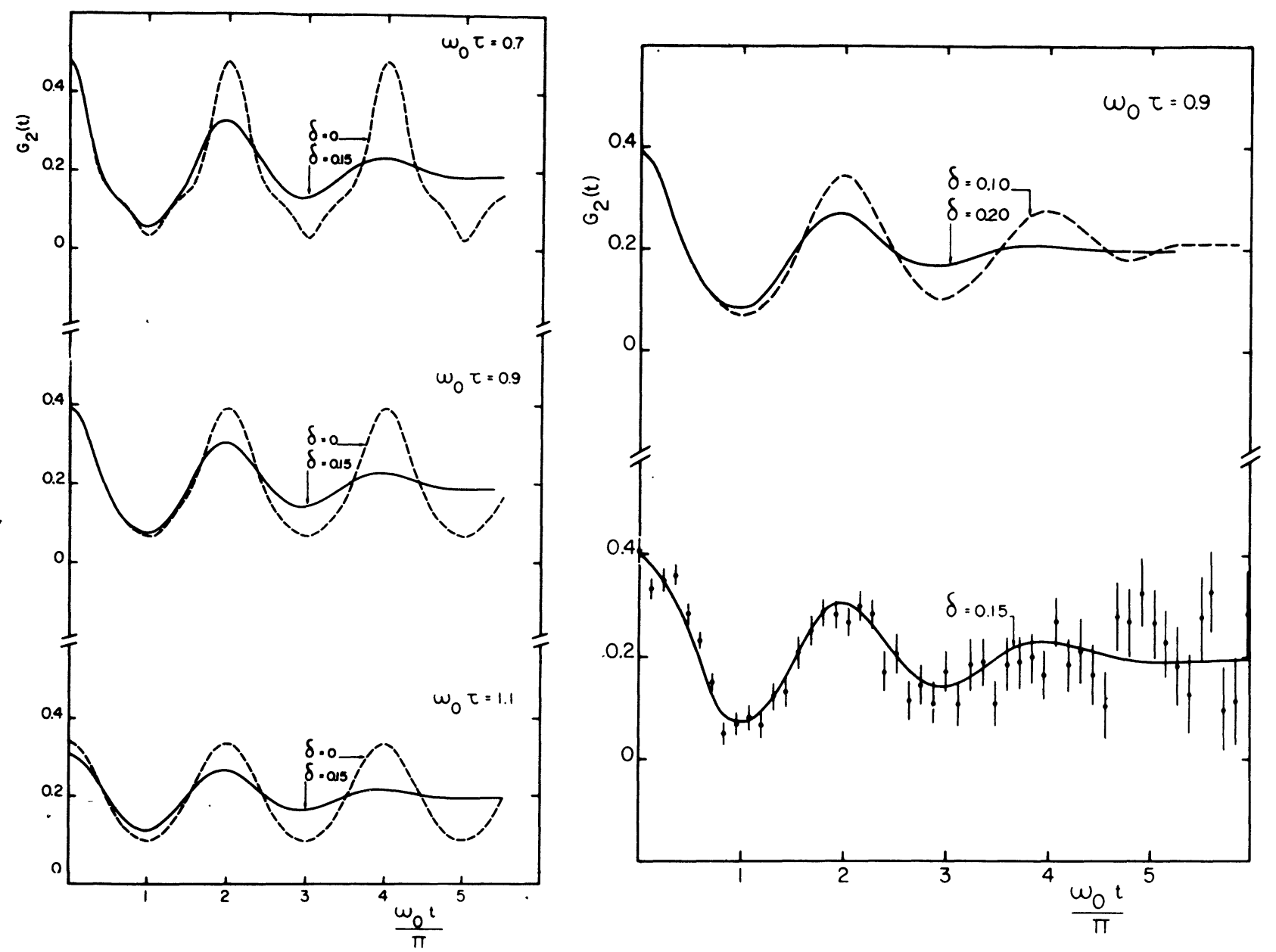

FIG. 13. - TDPAC static quadrupole hfi measurement on 36 ns-state in ${ }^{169} \mathrm{Tm}$ [34] (see text). Experimental result is compared to various curves calculated for a $7 / 2$-spin $\left(\omega_{0}\right.$ is the average quadrupole interaction; $\tau$ is the time resolution $-4.7 \mathrm{~ns}$ in this experiment; $\delta$ is the spread around $\omega_{0}$ ).

excellent agreement with a resistivity measurement on quenched pure $\mathrm{Ni}$ [36] ; (iii) in an unpublished latticelocation experiment performed on implanted $\mathrm{FeAu}$ by the authors of Ref. [33], the backscattering yield from $\mathrm{Au}$ was found to increase from 0.85 at room temperature [37] to 1.00 after annealing at $450{ }^{\circ} \mathrm{C}$. Hence, the transition (Fig. 12) that entails precipitation of insoluble $\mathrm{Yb}$ in $\mathrm{Fe}$ has the reverse effect on the soluble $\mathrm{Au}$ impurities : the thermal energy is high enough to dissociate these from the vacancy clusters that trapped them.

4. Conclusion. - More complete descriptions of these post-implantation impurity-defect complexes will certainly follow in the near future. For the moment, their existence and their influence on impurity behaviour have been established in isolated cases, and some of their properties are reasonably well understood. Unfortunately, room temperature implantations give rise to complex phenomena and most cases studied so far are probably too complicated to be of basic interest for radiation damage studies per se. One would like to combine the techniques of table II down at temperatures where single vacancies or interstitials interact with the implanted impurities [32]. Some programs have developed along this line for insulators or semiconductors, but considerable experimental work remains to be done for normal and magnetic metals. Conversely, higher-than-room temperature implantations may be of advantage to study defect kinetics [17] and possibly the electronic features of impurity defect interactions when size effects are not too drastic. As things stand today, considering the increasing efforts spent on the subject, it seems likely that the matrix of table II will open the door to many new basic aspects of radiation damage problems. Incidently, the above discussion implies that the phenomena described in $\S 3$ could well affect alloys prepared by methods other than ion-implantation, such as high-temperature quenching or « splatcooling $"$.

From the hfi experimentalists' point of view, it is doubtful whether the results obtained so far can lead to a universal recipe for avoiding radiation damage hazards when setting out to use an ion-implanted radioactive source. They do, however, strongly suggest 
that in all cases one should be prepared to justify in detail that, if an experiment is designed to measure some quantity such as a hyperfine field or a nuclear $g$-factor, it is insensitive to radiation damage effects or that they have been carefully taken into account. With this restriction in mind, the use of ion-implantation and hfi techniques offers a unique possibility of studying-besides electromagnetic nuclear properties, radiation damage and the microscopic properties of dilute alloys - a wide variety of fascinating problems, from surface physics and impurity states in superconductors to mineralogy via simulations of solar wind implantation : it is certainly not an idle field !

Acknowledgments. - It is a pleasure to thank Prof. J. Friedel, Dr. P. Lucasson, and Dr. P. Vajda for discussions and comments. Most of the ideas in Part 3 of this paper grew out of work with F. Abel, M. Bruneaux, C. Cohen, J. Chaumont, J. Flouquet and L. Thomé. The errors, however, are my own.

\section{Bibliographie}

[1] A general introduction to hfi may be found in Hyperfine Interactions, ed. Freeman, A. J., and Frankel, R. B. (Academic Press, N. Y.) (1967).

[2] Wertheim, G. K., The Mössbauer Effect (Academic Press, N. Y.) (1964).

[3] On nuclear orientation and perturbed angular correlations, see the reviews by DE GROOT, S. R. and HUISKAMP, W., StFFFen, R. M. and Frauenfelder, H., Alpha-, beta-, and gamma-ray spectroscopy, Chap. XIX (North Holland, Amsterdam) (1964) p. 271. For a discussion of recent nuclear orientation studies of dilute alloys see Flouquet, J., Annls. de Phys. 8 (1973) 5.

[4] Hyperfine Structure and Nuclear Radiation, ed. Matthias (E.) and Shirley (D. A.) (North Holland, Amsterdam) (1968).

[5] Discussion Meeting on Ion Implantation and Hyperfine Interactions, Proc. R. Soc. A 311 (1969) 1-209.

[6] Hyperfine Interactions in Excited Nuclear States, ed. Goldring (G.) and Kalish (R.) (Gordon and Breach, N. Y.) (1971) p. 291.

[7] Perturbed Angular Correlations in Nuclear Desintegrations, ed. Van Nooijen (B.) and Van Krugten (H.) (Rotterdam University Press, Rotterdam) (1971).

[8] Czuzek, G., Centennial Meeting of the French Physical Society, Vittel May 1973, J. Physique, 34 (1974).

[9] Lindhard, J. and Winther, A., Nucl. Phys. A 166 (1971) 416.

[10] Abragam, A. and Pound, R. V., Phys. Rev. 92 (1953) 943. Gabriel, H. and Bosse, J., in Ref. [7].

Blume, M. and Clauser, M. J., Phys. Rev. B 3 (1971) 583.

Hartmann-Boutron, F. and Spanjaard, D., J. Physique, 30 (1969) 975.

[11] Narath, A., CRC Crit. Rev. Sci. 3 (1972) 1.

[12] De WaARd, H., Proc. IAEA Conf. on Appl. Mössbauer Effect (1972), p. 123. See also : INIA, P. and DE WAARD, H., ref. [7], and INIA, P., Thesis. Univ, of Gröningen (1971).

[13] Proc. Int. Conf. on Nucl. Moments and Nucl. Structure, J. Phys. Soc. Japan 34 (Suppl.), 1973.

[14] Colloquium on Static Properties of Nuclei, Centennial Meeting of the French Physical Society, Vittel, May 1973, J. Physique 34 (1973) C4.

[15] Cameron, J. A., Gardner, P., Prestwich, W., Zamori, Z. and Santry, D. C., Can. J. Phys. 48 (1970) 2725.

[16] HaAs, H., Leitz, W., Mahnke, H., Semmler, R., Sielemann, (R.) and Wichert, Th., Phys. Rev. Lett. 30 (1973) 656.

[17] Bertschat, H., Christiansen, J., Mahnke, H., RecknaGel. E., Scatz, G., SielemanN, R. and Wittuhn, W., Phys. Rev. Lett. 25 (1970) 102.

[18] Sugimoto, K., Nakai, K., Matuda, K. and Minamisono, T., J. Phys. Soc. Japan, 25 (1968) 1258.
[19] Grodzins, L., Brochers, R. and Hagemann, G., Phys. Lett. 21 (1966) 214.

Boehm, F., Hagemann, G. and Winther, A., ibid. (1966) 21217.

[20] Bernas, H. and Gabriel, H., Phys. Rev. B 7 (1973) 468.

[21] Gebbie, D. W., Scherer, C., Huber, D., Heestand, G. M. and Borchers, R., Phys. Rev. B 7 (1973) 4821.

[22] SpanjaArd, D., Ph, D. Thesis, Orsay 1972, and SpaAnjard, D., MARSH, J. D. and StONE, N. J., to be published in J. Phys. (London).

[23] E. g. : QUÉRÉ, Y., Défauts Ponctuels dans les Métaux (ed. Masson), Paris 1967 and ref. therein. Other useful introductions include Dienes, G. J., in Proc. Cairo Solid State Conf. ed. A. Bishay (Gordon and Breach, N. Y.) (1967) and FrIEDEL, J. in Interactions of Radiation with Solids, ed. R. Strumane (North Holland) (1964).

For Au, see DaOud, M., Thesis, Paris (1972) and DAOUD, M. et al. Phys. Lett. 42A (1972) 169.

[24] Vacancies and Interstitials in Metals, ed. Seeger (A.) (North Holland Amsterdam) (1970) and references therein.

[25] See for example Ion Implantation in Semiconductors, MaYer, J. W., ERIKsson, L. and Davies, J. A. (Academic Press, New York) (1970).

[26] Benoît, A., Flouguet, J. and SAnchez, J., to be published in Phys. Rev. B.

[27] Krane, K., Olsen, C. E. and Steyert, W. A., Nucl. Phys. A 197 (1972) 352.

[28] Bernas, H., Ruault, M. O. and Jouffrey, B., Phys. Rev. Lett. 27 (1971) 859.

[28b] Ruault, M. O., Jouffrey, B., Chaumont, J. and Bernas, H., Int. Conf. Appl. Ion Beams to Metals, Albuquerque, October 1973, to be published.

[29] Doyama, M. and Koehler, J., Phys. Rev. 127 (1962) 21.

[30] Behar, M. and Steffen, R. M., Phys. Rev. Lett. 29 (1972) 116 and Phys. Rev. 7 (1973) 788.

[31] Janot, C. and Gibert, H., Phil. Mag. 27 (1973) 545.

[32] Mansel, W., Vogl, G. and КосH, W., Phys. Rev. Lett. 31 (1973) 359.

[33] Abel, F., Bruneaux, M., Cohen, C., Chaumont, J., Thomé, L. and Bernas, H., Solid State Commun. 13 (1973) 113.

[34] Glaeser, W. and Wever, H., Phys. Stat. Sol. 35 (1969) 367.

[35] Feldman, L. C., Kaufmann, E., Mingay, D. W. and Augustyniak, W., Phys. Rev. Lett. 27 (1971), 1145.

[36] Scherrer, S. and Deviot, B., J. Physique 33 (1972) 895. The zone-melted samples used in this work came from the same source (C. E. N.-Grenoble) as those used in [33].

[37] This value is in agreement with the result of AleXander, R., Poate. J. and Morgan, D. V., ref. [6] p. 229. 